\title{
Butea superba-Induced Amelioration of Cognitive and Emotional Deficits in Olfactory Bulbectomized Mice and Putative Mechanisms Underlying Its Actions
}

\author{
Daishu Mizuki ${ }^{1}$, Zhao Qi $^{1}$, Ken Tanaka ${ }^{1}$, Hironori Fujiwara ${ }^{1}$, Tsutomu Ishikawa ${ }^{2}$, Yoshihiro Higuchi ${ }^{3}$, \\ and Kinzo Matsumoto ${ }^{1, *}$ \\ ${ }^{1}$ Institute of Natural Medicine, University of Toyama, 2630 Sugitani, Toyama-shi, Toyama 930-0194, Japan \\ ${ }^{2}$ Graduate School of Pharmaceutical Sciences, Chiba University, 1-8-1 Inohana, Chuo-ku, Chiba 260-8675, Japan \\ ${ }^{3}$ Material Development Laboratories, Shiratori Pharmaceutical Co., Ltd., 6-11-24 Tsudanuma, Narashino, \\ Chiba 275-0016, Japan
}

Received November 28, 2013; Accepted February 4, 2014

\begin{abstract}
This study investigated the effects of alcoholic extract of Butea superba (BS) on cognitive deficits and depression-related behavior using olfactory bulbectomized (OBX) mice and the underlying molecular mechanisms of its actions. OBX mice were treated daily with BS (100 and $300 \mathrm{mg} / \mathrm{kg}$, p.o.) or reference drugs, tacrine $(2.5 \mathrm{mg} / \mathrm{kg}$, i.p.) and imipramine (10 mg/kg, i.p.) from day 3 after OBX. OBX impaired non-spatial and spatial cognitive performances, which were elucidated by the novel object recognition test and modified Y maze test, respectively. These deficits were attenuated by tacrine and BS but not imipramine. OBX animals exhibited depressionlike behavior in the tail suspension test in a manner reversible by imipramine and BS but not tacrine. OBX down-regulated phosphorylation of synaptic plasticity-related signaling proteins: NMDA receptor, AMPA receptor, calmodulin-dependent kinase II, and cyclic AMP-responsive element-binding protein. OBX also reduced choline acetyltransferase in the hippocampus. BS and tacrine reversed these neurochemical alterations. Moreover, BS inhibited ex vivo activity of acetylcholinesterase in the brain. These results indicate that BS ameliorates not only cognition dysfunction via normalizing synaptic plasticity-related signaling and facilitating central cholinergic systems but also depression-like behavior via a mechanism differing from that implicated in BS amelioration of cognitive function in OBX animals.
\end{abstract}

Keywords: Butea superba, olfactory bulbectomy, cognitive and emotional deficit, synaptic plasticity-related signaling, cholinergic system

\section{Introduction}

Butea superba (BS) (Red Kwao Kua in Thai) is an herb in the family Papilionaceae (Leguminosae) which is abundantly distributed in Thai deciduous forests. The tuberous roots of this plant have long been taken as a folk medicine not only to increase physical and mental strength but also to prevent aging-related symptoms such as impaired sexual performance in middle-aged or elderly males $(1,2)$. Evidence from a randomized double-

*Corresponding author. mkinzo@inm.u-toyama.ac.jp

Published online in J-STAGE on March 19, 2014

doi: 10.1254/jphs.13252FP blind clinical trial (3) showed that oral administration of powdered tubers of this plant to patients with erectile dysfunction (ED), aged 30 to 70 years, caused noticeable improvement without apparent toxicity. In fact, this clinical finding was supported by pharmacological (2) and chemical studies of this plant (4), demonstrating that BS enhances penile erection in rats and that some isoflavonolignans such as butesuperin $\mathrm{A}$ and $\mathrm{B}$ are chemical constituents with inhibitory activities against phosphodiesterase type 3A and type 5, enzymes targeted for ED therapy. Its ethno-medical uses and chemical constituents suggest that BS may have potential for the treatment of neurocognitive and/or neuropsychiatric symptoms. 
Alzheimer's disease (AD) is a progressive neurodegenerative disorder that is characterized by memory dysfunction and behavioral and psychological symptoms of dementia (BPSD), including depression. An increasing population of $\mathrm{AD}$ patients is a serious social and economic problem in super-aging societies worldwide (5). Current approved treatment with anti-dementia drugs such as donepezil, an acetylcholinesterase inhibitors, and memantine, an $N$-methyl-D-aspartate receptor (NMDA) antagonist, has provided marginal therapeutic benefits without affecting the progression of the disease (6). Therefore, new drug discovery and the establishment of new therapeutic methods are greatly needed.

Olfactory bulbectomy (OBX) in rodents has been widely used as an animal model to investigate emotional dysfunction such as depression (7-10). Indeed, OBX causes depression-like behavioral and neurochemical alterations in rodents that are susceptible to antidepressant treatment $(11-13)$. Moreover, OBX has been used as one of the $\mathrm{AD}$ models since impairment of olfactory perceptual acuity is observed not only at the early stage of $\operatorname{AD}(14,15)$ but also in a transgenic AD model of mice over-expressing a mutant form of the human amyloid- $\beta$ precursor protein (16). In addition, OBX causes elevation of the $\mathrm{A} \beta$ level in the brain (17) and the degeneration of septo-hippocampal cholinergic system in rodents $(8,9$, $18)$, and thereby induces cognitive dysfunction $(8-10)$.

In this study, we elucidated the anti-dementia and antidepressant drug-like effects of BS and the mechanism underlying its action using an OBX model mouse to obtain a better understanding of the potential availability of BS for the treatment of cognitive and emotional dysfunction. The present findings clearly demonstrated that BS ameliorates cognitive and depression-like emotional deficits of OBX mice and that the effects on the cognitive deficits are mediated in part by normalization of OBX- induced dysfunction of neuroplasticity-related neuronal signaling and cholinergic systems. Our findings suggested that $\mathrm{BS}$ is useful for the treatment of $\mathrm{AD}$ patients with depressive symptoms.

\section{Materials and Methods}

\section{Animals}

This study was conducted according to the experimental protocols as described in Fig. 1. A total of 85 male ddY mice were obtained at the age of 8 weeks (Japan SLC, Inc., Shizuoka). The animals were habituated to the laboratory animal room for at least 1 week before surgery. Food and water were available ad libitum. Housing was thermostatically maintained at $24^{\circ} \mathrm{C} \pm 1{ }^{\circ} \mathrm{C}$ with constant humidity (65\%) and a 12-h light-dark cycle (lights on: 07:00 - 19:00). The behavioral experiments were performed during the light phase from 9:00 to 18:00. The present studies were conducted in accordance with the Guiding Principles (NIH publication \#85-23, revised in 1985) for the Care and Use of Animals and were approved by the Institutional Animal Use and Care Committee of the University of Toyama.

\section{Preparation of plant extract}

The ethanol extract of BS was prepared as previously described. Briefly, the root of BS was collected in Lampang Province in Thailand in 2011 and identified by Dr. Chaiyo Chaichantipyuth (Department of Pharmacognosy, Faculty of Pharmaceutical Sciences, Chulalongkorn University, Thailand). The dried powder of the root $(52 \mathrm{~g})$ was extracted with $200 \mathrm{ml}$ of $95 \%$ ethanol at $75^{\circ} \mathrm{C}$ for $2 \mathrm{~h}$ and filtrated. This step was repeated 3 times; and the filtrated samples were combined, concentrated under reduced pressure at $40^{\circ} \mathrm{C}$, and then dried in vacuo. The yield of the extraction from the dried root was calculated



$\left\{\begin{array}{l}\text { a) Object recognition test } \\ \text { b) Modified Y-maze test } \\ \text { c) Tail suspension test }\end{array}\right.$

Fig. 1. Schematic drawing of experimental protocol. ddY mice received daily administration of Butea superba extract (BS; $100-300 \mathrm{mg} / \mathrm{kg}$, p.o.), tacrine (THA; $2.5 \mathrm{mg} / \mathrm{kg}$ per day, i.p.), imipramine (IMP; $10 \mathrm{mg} / \mathrm{kg}$ per day, i.p.) or tap water from day 3 after the operation. The administration was repeated during the experimental period. Each experiment was conducted at $1-2$-week interval as described in the text. After completing the behavioral experiments, the mice were decapitated under pentobarbital anesthesia. The brain tissues were used for neurochemical studies. 
as $8.9 \%(\mathrm{w} / \mathrm{w})$. The BS (voucher specimen No. TMPW27997) and its extract (voucher specimen no INM-531) used in this study were deposited at our institute.

LC-MS analyses were also performed with a Shimadzu LC-IT-TOF mass spectrometer equipped with an ESI interface. The ESI parameters were as follows: source voltage, $+4.5 \mathrm{kV}$; capillary temperature, $200^{\circ} \mathrm{C}$; and nebulizer gas, $1.5 \mathrm{l} / \mathrm{min}$. The mass spectrometer was operated in positive ion mode scanning from $\mathrm{m} / \mathrm{z}$ 200 to 2000. A Waters Atlantis T3 column $(2.1 \mathrm{~mm}$ i.d. $\times 150 \mathrm{~mm}$ ) was used and the column temperature was maintained at $40^{\circ} \mathrm{C}$. The mobile phase was a binary eluent of A) $5 \mathrm{mM}$ ammonium acetate solution and B) acetonitrile under the following gradient conditions: $0-30 \mathrm{~min}$, linear gradient from $10 \%$ to $100 \% \mathrm{~B}$ and $30-40 \mathrm{~min}$, isocratic at $100 \% \mathrm{~B}$. The flow rate was $0.2 \mathrm{ml} / \mathrm{min}$. Mass spectrometry data obtained from the extract have been listed in the MassBank database (19) and stored together with the pharmacological information on the extract in the Wakan-Yaku DataBase system (http://wakandb.u-toyama.ac.jp/wiki/LCMS:Butea INM-531), Institute of Natural Medicine, University of Toyama.

\section{Surgical operation}

OBX of mice was conducted on day 0 as previously described $(8-10)$. The mice were anesthetized with sodium pentobarbital $(60 \mathrm{mg} / \mathrm{kg}$, i.p. $)$ and fixed on stereotactic instruments (Narishige, Tokyo). With 1\% lidocaine solution used as a local anesthetic, the skull covering the bulbs was exposed by skin incision, and then a 1-mm burr hole was drilled. The bilateral bulbs were aspired through a syringe and the cavity of the bulbs was filled with a hemostatic gelatin sponge. After completing the behavioral studies, all the animals were sacrificed and the operated lesion was verified visually. The data from animals with less than $70 \%$ removal or with no intact cortex were excluded from the data analysis. The success rate of surgical operation was $98 \%$ (49/50). Sham operation was performed in a similar way without removal of the bulb. At the end of the experiments, the olfactory bulbs of the sham-group mice were also confirmed to be intact.

\section{Drug administration}

Either vehicle water or test drugs were administered daily from day 3 after surgery during the experimental period. On the behavioral testing day, administration was conducted $1 \mathrm{~h}$ before the testing. Sham-group mice and OBX-control group mice were perorally administered water. Tacrine (9-amino-1,2,3,4-tetrahydro-acridine $\mathrm{HCl}$ ) and imipramine $\mathrm{HCl}$ (Sigma-Aldrich Co., St. Louis, MO, USA), reference standard drugs, were dissolved in $0.9 \%$ saline and administered once daily at doses of 2.5 and $10 \mathrm{mg} / \mathrm{kg}$ (i.p.), respectively. BS extract was suspended in water and given perorally at daily doses equivalent to 100 and $300 \mathrm{mg}$ (dried herb weight) $/ \mathrm{kg}$. The daily doses of BS used in the present animal experiments were almost 10 - 30 times more than that used for human ED therapy (3).

\section{Behavioral study}

The following behavioral experiments were started from 2 weeks after surgery.

\section{Novel object recognition test (ORT)}

ORT was conducted at days $17-20$. This test is based on the tendency of mice to discriminate a familiar from a new object. This test was conducted as previously described $(8,9,20)$ with minor modification. Briefly, mice were individually habituated to an observation box $(50 \times 50 \times 40 \mathrm{~cm})$ for $10 \mathrm{~min}$ on the day before the experiment. The ORT consisted of a sample phase trial and a test phase trial. In the sample phase trial, each mouse was placed in the observation box where two identical objects, object 1 and object 2 , were placed separately, and allowed to explore the area freely for $5 \mathrm{~min}$. The total time that the mouse spent exploring each of the two objects was measured and then the mouse was returned to the home cage. In the test phase trial performed $30 \mathrm{~min}$ after the sample phase trial, one of the two objects was replaced by an identical copy (familiar object) and the other by a novel object. Performance of the animals in this test was video-recorded for later analysis. In these trials, the exploration of an object was defined as directing the nose to the object at a distance of $<2 \mathrm{~cm}$ according to previous reports $(8,9$, 20 ) and the time spent exploring each of the two objects was analyzed using SMARTW ver. 2.5 (PanLab, S.L., Barcelona, Spain) with a tri-wise module to detect the head, center mass, and base tail.

\section{Modified Y-maze test}

A modified version of the Y-maze test was conducted at days 24-27 according to Yamada et al. (9). The apparatus used for this test consists of black polypropylene walls with 3 arms, each 40-cm-long, 12-cm-wide at the top, 3-cm-wide at the bottom, and 18-cm-high. This test was a two-trial task with a sample phase trial and a test phase trial that were separated by an inter-trial interval. In the sample phase trial, each mouse was individually placed in the maze with one of the $3 \mathrm{arms}$ closed. The animal was allowed to explore the other 2 arms freely for $5 \mathrm{~min}$. Thirty minutes after the sample phase trial, the animal was again placed in the maze with all 3 arms opened, and allowed to explore the arms 
freely. The previously closed arm that was opened in the test phase trial was defined as the new arm. The animal behavior was video-recorded for later analysis. Percent time spent in the new arm was analyzed using SMART ${ }^{\circledR}$ system ver. 2.5 (PanLab).

\section{Tail suspension test (TST)}

We employed TST to assess the antidepressant effects of the test drugs (21). This test was conducted at days $38-40$ as previously described (10). Briefly, mice were subjected to the short-term inescapable stress of being suspended by the tail, which leads to the development of an immobile posture. The animals were separately suspended $50 \mathrm{~cm}$ above the floor in a chamber by adhesive tape placed approximately $2 \mathrm{~cm}$ from the tip of the tail. The animal behavior in the test was videorecorded for later analysis. Immobility was defined as a state with movement speed no more than $0.05 \mathrm{~cm}^{2} / \mathrm{s}$ using SMART ${ }^{\circledR}$ system ver. 2.5 ; immobility time was recorded for $8 \mathrm{~min}$ and the performance during the last 6-min period was analyzed.

\section{Neurochemical study}

One week after completing the behavioral experiments, mice were decapitated under pentobarbital $(50 \mathrm{mg} / \mathrm{kg}$, i.p.) anesthesia. The hippocampal tissues were quickly dissected out and stored at $-80^{\circ} \mathrm{C}$ until use.

\section{Western blotting}

Expression of synaptic plasticity-related proteins in the hippocampus was analyzed using western blotting as previously described $(8,22,23)$. The following primary antibodies were used: anti-NMDAR1 rabbit polyclonal antibody (1:1000 dilution), anti-phospho-NMDAR1 (p-NMDAR1) (pSer896) rabbit polyclonal antibody (1:1000 dilution) (Cell Signaling Technology, Danvers, MA, USA); anti-glutamate receptor 1 (GluR1) rabbit polyclonal antibody (1:1000 dilution), anti-phoshoGluR1 (p-GluR1) (pSer 831) rabbit polyclonal antibody (1:1000 dilution) (Sigma-Aldrich); anti-CaMKII $\alpha$ (A-1: sc-13141) mouse monoclonal antibody (1:1000 dilution), anti-phospho-CaMKII (p-CaMKII) (pThr286) rabbit polyclonal antibody (1:1000 dilution), anti-CREB (48H2) rabbit monoclonal antibody (1:1000 dilution), antiphospho-CREB (p-CREB) (pSer133) rabbit monoclonal antibody (1:1000 dilution), anti-choline acetyltransferase (ChAT) goat polyclonal antibody (1:5000 dilution) (AB-144P; Millipore, Temecula, CA, USA); and anti- $\beta$ actin mouse monoclonal antibody (1:10,000 dilution, Abcam $^{\circledR}$, Cambridge, UK). The immune complexes were detected by the enhanced chemiluminescence method (ImmobilonTM Western Chemiluminescent HRP Substrate) (Millipore) and imaged using Lumino Image
Analyzer LAS-4000R (Fuji Film, Tokyo). The quantity of immune-reactive bands was analyzed using ImageQuant TL software (GE Healthcare, Buckinghamshire, UK). The quantity of immunoreactive bands was normalized by comparison with their expression levels in treatment-naïve control mice. The expression levels of each target protein were reprobed using a Blot Restore Membrane Rejuvenation Kit (Millipore).

\section{Ex vivo measurements of acetylcholinesterase (AChE)} activity in the brain

Ex vivo AChE activity in the cerebral cortex was determined by the colorimetric method as previously described $(8-10,24)$. Briefly, 9-week-old male mice received daily administration of $300 \mathrm{mg} / \mathrm{kg} \mathrm{BM}$ for 14 days. The animals were decapitated $1 \mathrm{~h}$ after the last administration and the cerebral cortices were dissected out from the brain. The frozen cortex was weighed and homogenized in 10 volumes of $0.1 \mathrm{M}$ phosphate buffer (pH 7.4) containing 1\% Triton-X-100. After centrifugation at $15,000 \times g$ and $4^{\circ} \mathrm{C}$ for $20 \mathrm{~min}$, the clear supernatants were collected and served as the enzyme source. Cholinesterase activity was determined in $50 \mu \mathrm{l}$ aliquots of homogenates (run as duplicates) in a 96-well flatbottomed microplate. The reaction was started by

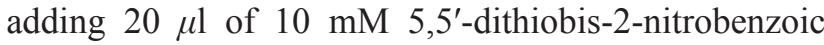
acid (DTNB), $20 \mu \mathrm{l}$ of $7.5 \mathrm{mM}$ acetylthiocholine (ATCI), and $160 \mu \mathrm{l}$ of $0.1 \mathrm{M}$ sodium phosphate buffer ( $\mathrm{pH} 8.0$ ). The spectrophotometric absorption at $405 \mathrm{~nm}$ during a 5 -min incubation period at $25^{\circ} \mathrm{C}$ was quantitatively measured using a microplate reader (Sunrise Classic; TECAN Japan, Kawasaki) and is expressed as nmol ACh hydrolyzed/min per mg tissue.

\section{Data analyses}

The data are expressed as the mean \pm S.E.M. The data obtained from the object recognition test were analyzed by the paired Student's $t$-test and the data obtained from other behavioral and neurochemical experiments were analyzed by one-way analysis of variance (ANOVA) followed by a post hoc multiple comparison test (StudentNewman-Keuls method) as appropriate. Differences of $P<0.05$ were considered significant. The analysis was conducted using SigmaStat ${ }^{\circledR}$ ver. 3.5 (SYSTAT Software Inc., Richmond, CA, USA).

\section{Results}

$B S$ attenuates $O B X$-induced non spatial short-term memory deficits in the object recognition test

The object recognition test was used to evaluate the effects of BS on the non-spatial cognitive memory of OBX mice (25). In the sample phase trials, none of the 


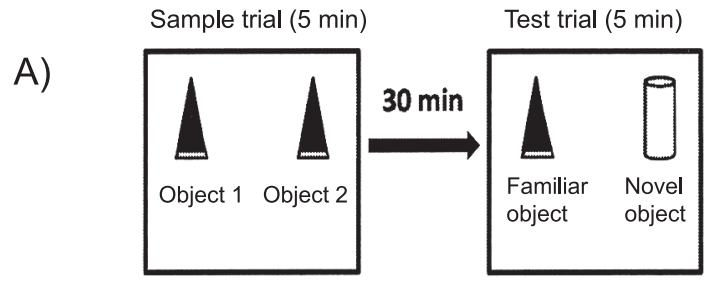

B)

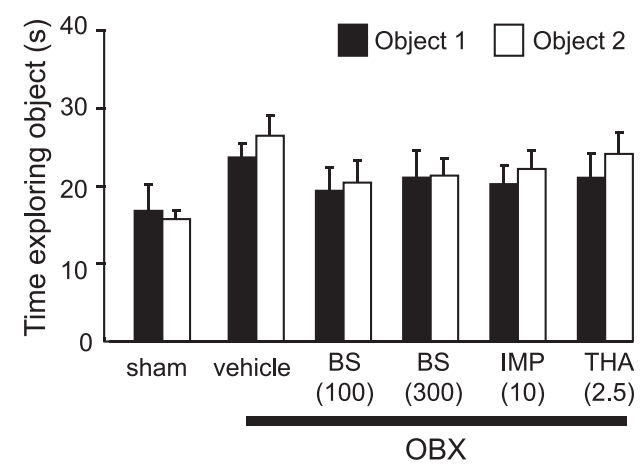

C)

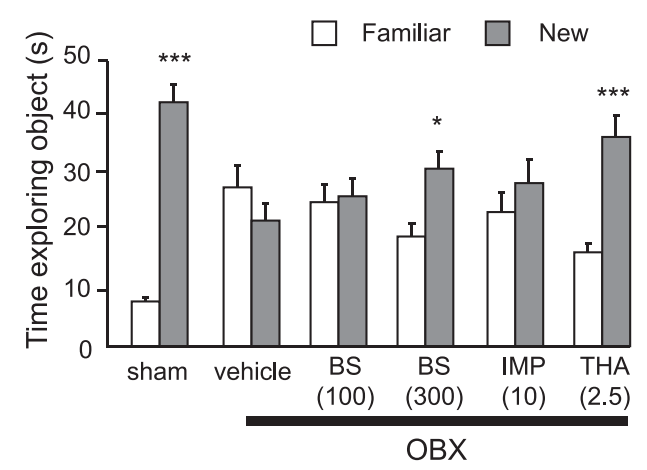

Fig. 2. Treatment of BS (300 mg/kg) and tacrine (THA) improves object recognition memory deficit in OBX mice. A: Experimental protocols depicting the novel object recognition test (ORT). B and C: The sample phase (B) and the test phase trials (C) were conducted at a 30 -min interval. Each data column represents the mean \pm S.E.M. $(\mathrm{n}=10) . * P<0.05$ and $* * * P<0.001$ vs. time spent exploring a familiar object (paired $t$-test).

animal groups showed significant differences in time spent exploring each identical object. In the test phase trial, the sham group spent a significantly longer time exploring the novel object than exploring the familiar one $(t=-10.500, \mathrm{df}=18, P<0.001)$, while the vehicletreated OBX group showed no preference for the novel object $(t=1.521, \mathrm{df}=18, P=0.163)$. BS $(300 \mathrm{mg} / \mathrm{kg}$ per day)- and tacrine-treated OBX mice, as well as the sham group, spent a significantly longer time exploring the novel object than the familiar one. (BS-treated OBX group: $t=-2.843, \mathrm{df}=18, P=0.019$; tacrine-treated OBX group: $t=-5.525, \mathrm{df}=18, P<0.001$ ) (Fig. 2). In contrast, BS (100 mg/kg per day)- and imipraminetreated OBX mice did not show a preference for the novel object.

\section{$B S$ treatment reverses spatial short-term memory deficits of OBX mice in the modified Y-maze test}

We also examined the effect of BS treatment on time visiting the novel arm in the modified Y-maze test. As shown in Fig. 3, the ratio of the time that the sham group spent visiting the novel arm to the time spent visiting the other two familiar arms was higher than the chance level of $33.3 \%$, indicating a preference for the novel arm over the familiar arms. The vehicle-treated OBX mice spent a significantly shorter time exploring the novel arm than the sham mice [one-way ANOVA: $\mathrm{F}(5,50)=8.420$,
A)

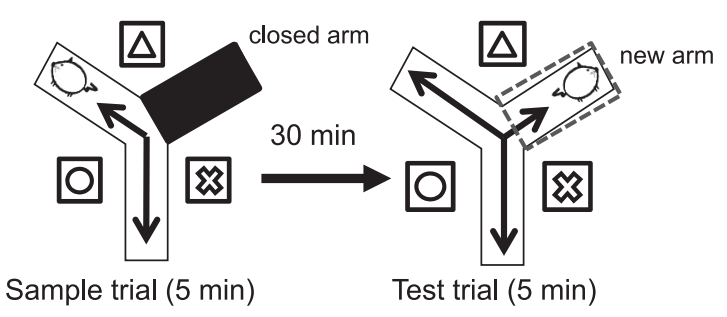

B)

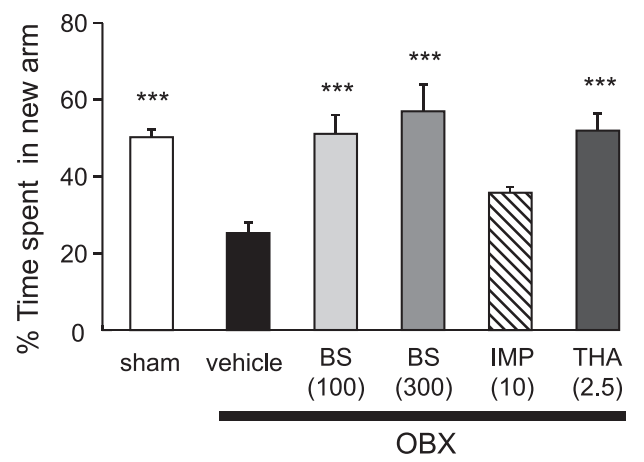

Fig. 3. Effects of BS and tacrine (THA) on OBX-induced spatial working memory deficit. A: Experimental protocols depicting the modified Y-maze test. The maze was surrounded by different spatial cues. The sample and test trials were conducted for 5 min at a 30-min interval. In the sample trial, each mouse was individually placed in the maze with one of the 3 arms closed. The previously closed arm that was opened in the test trial was defined as the novel arm. Results are expressed as \% time animals spent exploring the novel arm in the test trial. B: Spatial short-term working memory performance of sham and OBX groups in the test trials conducted after $30 \mathrm{~min}$. Each data column represents the mean \pm S.E.M. $(\mathrm{n}=9-12) . * * * P<0.001$ vs. vehicle-treated OBX group (post hoc Student-Newman-Keuls test). 
$P<0.001$; post hoc test: sham vs. vehicle-treated OBX, $P<0.001]$. BS $(100-300 \mathrm{mg} / \mathrm{kg}$ per day)- and tacrinetreated OBX mice spent a significantly longer time exploring the novel arm than the vehicle-treated OBX group [post hoc test: vehicle-treated OBX vs. BS $(100 \mathrm{mg} / \mathrm{kg}$ per day)-treated OBX, $P<0.001$; vehicletreated OBX vs. BS (300 mg/kg per day)-reated OBX, $P<0.001$; vehicle-treated OBX vs. tacrine-treated OBX, $P<0.001]$. On the other hand, imipramine administration had no effect on spatial working memory deficits in OBX mice [post hoc test: vehicle-treated OBX vs. imipramine-treated OBX, $P=0.096]$.

\section{$B S$ ameliorates depression-like behavior of OBX mice in the TST}

This test was used to evaluate OBX-induced depressive-like behavior. The vehicle-treated OBX mice showed a significantly longer immobility time than the sham group [one-way ANOVA: $\mathrm{F}(5,54)=4.303, P=$ 0.002; post hoc test: sham vs. vehicle-treated OBX, $P=0.006]$. BS ( $300 \mathrm{mg} / \mathrm{kg}$ per day)- and imipraminetreated OBX mice showed a significantly shorter immobility time than the vehicle-treated OBX mice [post hoc test: vehicle-treated OBX vs. BS $(300 \mathrm{mg} / \mathrm{kg}$ per day)-treated $\mathrm{OBX}, P=0.023$; vehicle-treated $\mathrm{OBX}$ vs. imipramine-treated OBX, $P=0.005$ ], whereas BS (100 $\mathrm{mg} / \mathrm{kg}$ per day) and tacrine treatment did not significantly reduce immobility time [post hoc test: vehicle-treated OBX vs. BS (100 mg/kg per day)-treated OBX, $P=0.069$; vehicle-treated OBX vs. tacrine-treated OBX, $P=0.204]$ (Fig. 4).

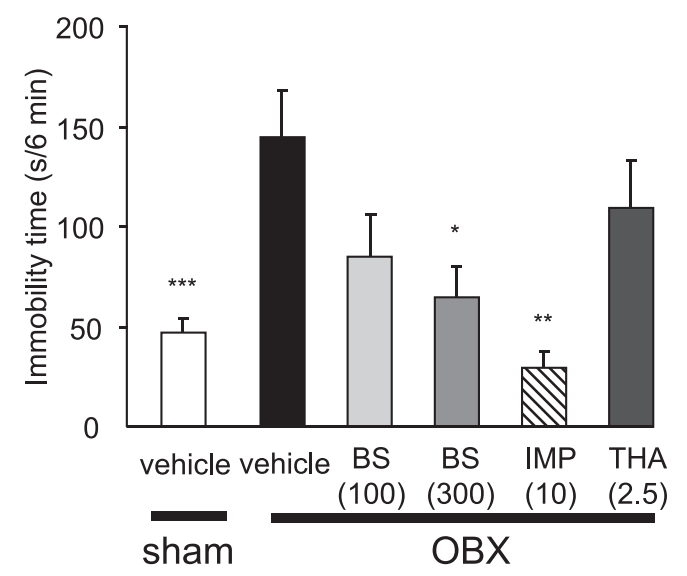

Fig. 4. Effects of BS, imipramine (IMP) and tacrine (THA) on OBX-induced depression-related behavior in the tail suspension test. Each data column represents the mean \pm S.E.M. $(\mathrm{n}=9-10)$. $* P<0.05, * * P<0.01$, and $* * * P<0.001$ vs. vehicle-treated OBX group (post hoc Student-Newman-Keuls test).
$B S$ reversed $O B X$-induced downregulation of glutamatergic neural plasticity in the hippocampus

To understand the molecular mechanisms underlying BS-induced improvement of cognitive deficits in OBX mice, we examined the effects of BS on the synaptic plasticity-related neuro-signaling pathway in the hippocampus of OBX mice by western blotting. No differences in the hippocampal NMDAR1 (NMDAR subunit), GluR1 (AMPAR subunit), CaMKII $\alpha$, and CREB levels were found among OBX, BS, or reference drugs (Fig. 5). The expression levels of phosphorylated NMDAR1, GluR1, CaMKII $\alpha$, and CREB in the OBX mice were significantly decreased compared with the levels in the sham mice. However compared with vehicle-treated sham mice, vehicle-treated OBX mice showed a significant decrease in the levels of pSer896-NMDAR1 [oneway ANOVA: $\mathrm{F}(4,15)=3.505, P=0.033$; post hoc test: sham vs. vehicle-treated OBX group, $P=0.039$ ], pSer831-GluR1 [one-way ANOVA: $\mathrm{F}(4,15)=5.863$, $P=0.005$; post hoc test: sham vs. vehicle-treated OBX group, $P=0.041$ ], pThr286-CaMKII [one-way ANOVA: $\mathrm{F}(4,15)=4.725, P=0.011$; post hoc test: sham vs. vehicle-treated OBX group, $P=0.010]$, and pSer133CREB [one-way ANOVA: $\mathrm{F}(4,15)=4.270, P=0.017$; post hoc test: sham vs. vehicle-treated OBX group, $P=0.032]$. BS and tacrine treatment ameliorated OBX-induced downregulation of pSer896-NMDAR1 [post hoc test: vehicle-treated OBX group vs. BS-treated OBX group, $P=0.042$; vehicle-treated OBX group vs. tacrine-treated OBX group, $P=0.036]$ and pSer831GluR1 [post hoc test: vehicle-treated OBX vs. BS (300 $\mathrm{mg} / \mathrm{kg}$ per day)-treated $\mathrm{OBX}, P=0.046$; vehicle-treated OBX vs. tacrine-treated OBX, $P=0.021]$ and pSer133CREB [post hoc test: vehicle-treated OBX group vs. BS $(300 \mathrm{mg} / \mathrm{kg}$ per day)-treated OBX group, $P=0.025$; vehicle-treated OBX group vs. tacrine-treated OBX group, $P=0.011$ ] and pThr286-CaMKII [post hoc test: vehicle-treated OBX vs. BS (300 mg/kg per day)-treated OBX, $P=0.017$; vehicle-treated OBX vs. tacrine-treated OBX, $P=0.012$ ] (Fig. 5: $\mathrm{B}-\mathrm{E}$ ). Imipramine treatment significantly reversed OBX-induced down-regulation of pThr286-CaMKII [post hoc test: vehicle-treated OBX vs. imipramine-treated OBX, $P=0.028]$ and pSer133CREB [post hoc test: vehicle-treated OBX vs. imipramine-treated OBX, $P=0.043$ ], but it had no effect on pSer896-NMDAR1 [post hoc test: vehicle-treated OBX vs. imipramine-treated $\mathrm{OBX}, P=0.103]$, pSer831GluR1 [post hoc test: vehicle-treated OBX vs. imipramine-treated OBX, $P=0.502]$.

$B S$ facilitated central cholinergic systems in the brain of OBX mice

The expression of ChAT, a cholinergic marker in the 

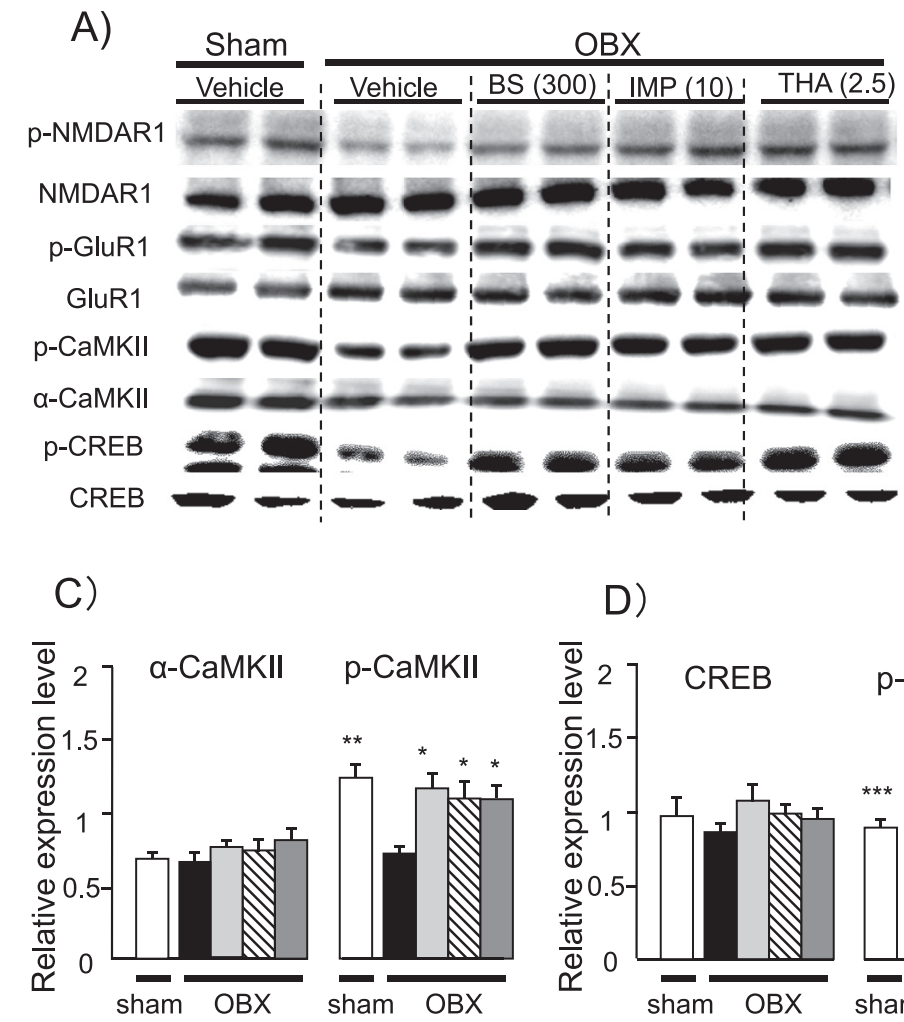

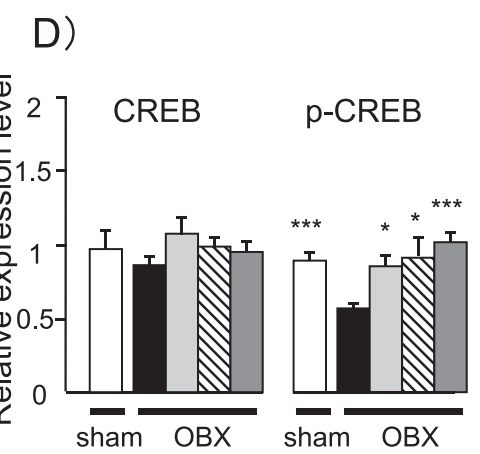

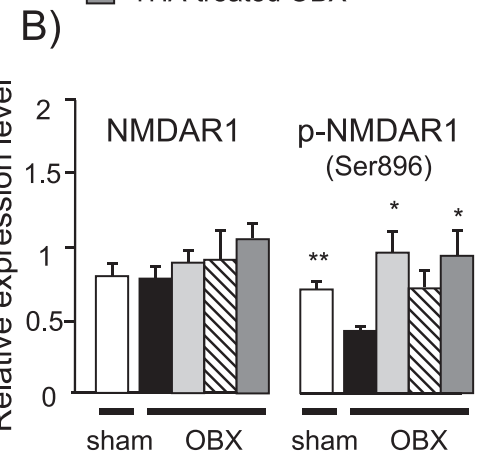

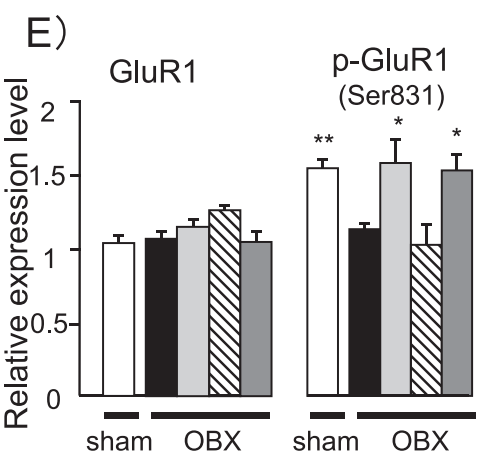

Fig. 5. Effects of BS and tacrine (THA) on synaptic plasticity-related signaling pathway in the hippocampus of OBX mice. A: Typical photos indicate expression levels of NMDAR1, p-NMDAR1 (p-Ser 896), GluR1, p-GluR1 (p-Ser831), CaMKII $\alpha$, p-CaMKII (p-Thr286), CREB, and p-CREB (pSer 133) in the hippocampi of sham and vehicle-, BS-, and THA-treated OBX animals. B - E: Quantitative comparisons of expression levels of p-NMDAR1/NMDAR1, p-GluR1 (p-Ser831)/GluR1, p-CaMKII/ CaMKII $\alpha$, and p-CREB (p-Ser133)/CREB. Each data column represents the mean \pm S.E.M. $(\mathrm{n}=4) . * P<0.05, * * P<0.01$, and *** $P<0.001$ vs. vehicle-treated OBX group (post hoc Student-Newman-Keuls test).

hippocampus, was examined by western blotting. Compared with that in the sham-operated group, the vehicletreated OBX group exhibited a reduced expression level of ChAT in the hippocampus [one-way ANOVA: $\mathrm{F}(4,15)=11.080, P<0.001$; post hoc test: sham vs. vehicle-treated OBX, $P=0.007]$. OBX-induced downregulation of ChAT expression was reversed by $\mathrm{BS}$ and tacrine but not by imipramine [post hoc test: vehicletreated OBX group vs. BS (300 mg/kg per day)-treated OBX group, $P=0.005$; vehicle-treated $\mathrm{OBX}$ group vs. tacrine-treated OBX group, $P<0.001$; vehicle-treated OBX group vs. imipramine-treated OBX group, $P=$ 0.087] (Fig. 6).

\section{The effect of tacrine and BS on ex vivo AChE activity in the brain}

We analyzed the effect of BS on ex vivo activities of $\mathrm{AChE}$ in the brain to examine the possible involvement of endogenous acetylcholine in the action of BS. As shown in Fig. 7, importantly, the activity of cortical $\mathrm{AChE}$ in the mice treated with $\mathrm{BS}$ and tacrine for 2 weeks was significantly lower than that in the vehicletreated mice [one-way ANOVA: $\mathrm{F}(2,19)=8.129$, $P=0.003$; post hoc test: vehicle-treated group vs. BS-treated group, $P=0.016$; vehicle-treated group vs. tacrine-treated group, $P=0.002]$.

\section{Discussion}

In this study, we investigated the effects of BS on OBX-induced cognitive deficits and depression-like behavior. The present findings demonstrated that BS administration ameliorates not only cognitive deficits but also depression-like behavior in OBX animals and suggested that the anti-dementia effect is at least in part due to restoring synaptic plasticity-related signaling and enhancement of cholinergic systems via inhibiting the activity of AChE in the brain. 


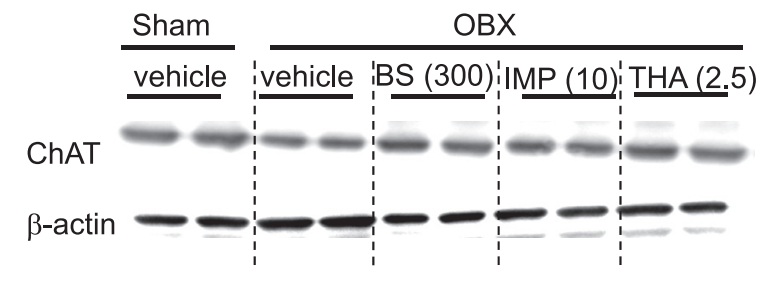

Choline acetyltransferase (ChAT)
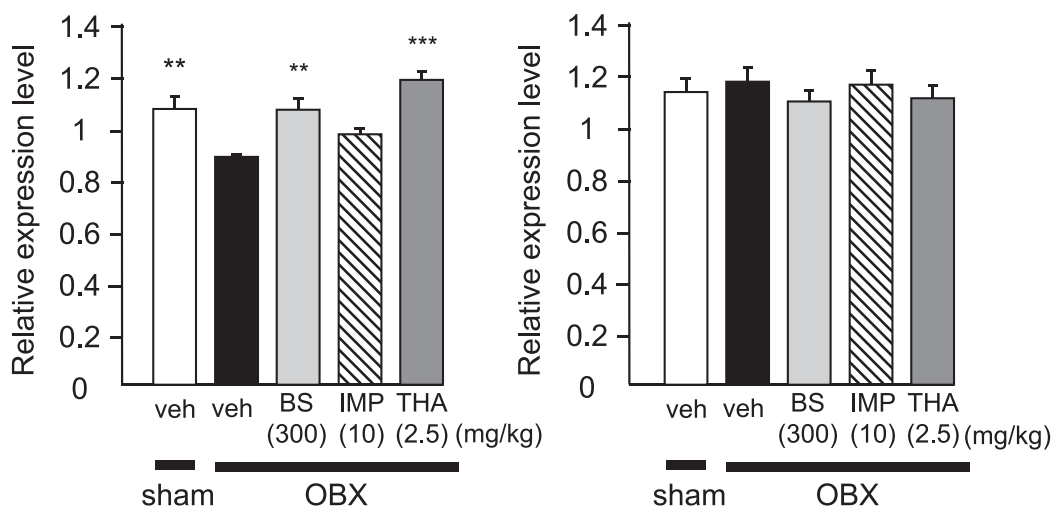

Fig. 6. BS and tacrine (THA) treatment reverses OBX-induced down-regulation of ChAT expression in the hippocampus. A: Typical photos indicating expression levels of ChAT. B: Quantitative comparisons of expression levels of ChAT. Each data column represents the mean \pm S.E.M. $(\mathrm{n}=4), * * P<0.01$ and $* * * P<0.001$ vs. vehicle-treated OBX group (post hoc Student-Newman-Keuls test).

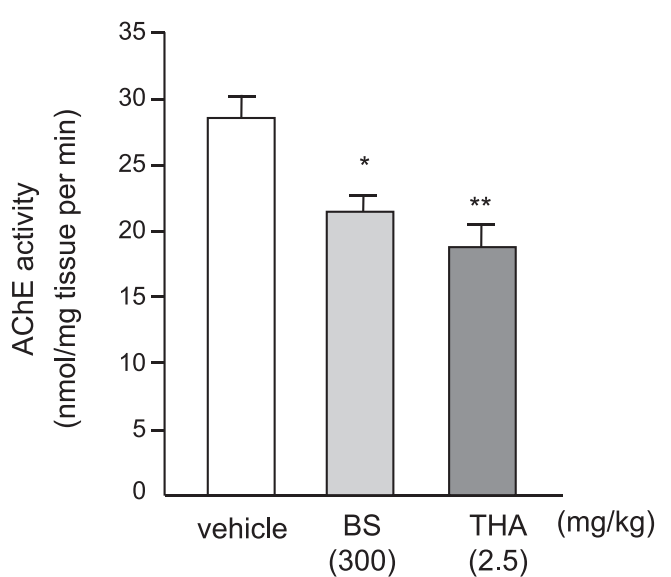

Fig. 7. Effects of BS and tacrine (THA) on the ex vivo acetylcholinesterase (AChE) activity in the cerebral cortices of mice. Nine-weekold male mice received daily administration of $300 \mathrm{mg} / \mathrm{kg}$ BM for 14 days. The animals were decapitated $1 \mathrm{~h}$ after the last administration and the cerebral cortices were dissected out from the brain. The activity of AChE was analyzed as described in the text. Each data column represents the mean \pm S.E.M. $(\mathrm{n}=7-8), * P<0.05$ and $* * P<0.01$ vs. vehicle-treated group (post hoc Student-Newman-Keuls test).

We first employed an object recognition test to elucidate short-term non-spatial working memory (26). Working memory is one of the types of short-term memory that have been reported to be impaired at an early stage in patients with AD (27). In the sample trial of this test, no significant difference in total time spent exploring two identical objects was observed between sham and OBX groups, indicating virtually no differences in the ability to recognize objects between animals.
In the test trial, sham-operated mice spent more time exploring a novel object, while vehicle-treated OBX mice failed to discriminate between familiar and novel objects, indicating impairment of non-spatial working memory. The result that OBX-induced impairment of object recognition performance was reversed by the AChE inhibitor tacrine agrees with our previous studies $(8,10)$ and supports the idea that OBX-induced impairment of object recognition performance involves central cholinergic dysfunction $(8,18)$.

Interestingly, BS treatment as well as tacrine could significantly ameliorate OBX-induced deficit of novel object recognition. The ameliorative effect of BS on the working memory deficit was further supported by the data obtained from the modified version of the $\mathrm{Y}$ maze test. Previous studies demonstrated that central cholinergic and glutamatergic systems are involved in short-term spatial working memory performance elucidated by the modified Y-maze test since the muscarinicreceptor antagonist scopolamine and the anti-NMDAreceptor antagonist MK-801 interfere with the performance in a manner reversible by AChE inhibitors $(9,28)$. In this study, we found that, consistent with previous reports $(8-10)$, OBX mice exhibited significantly impaired spatial working memory performance in the modified Y-maze test, and that the impairment was reversed by tacrine and BS but not by imipramine. These findings suggest that BS treatment can exhibit an antidementia effect like tacrine in OBX mice. Taken together with the data obtained using an object recognition test, it is likely that BS has the potential to improve both 
non-spatial and spatial working memory deficits.

We analyzed the expression levels of synaptic plasticity-related signaling proteins, a molecular biological feature of learning and memory $(8,29,30)$, to understand the mechanism underlying the effects of BS on the impaired cognitive performance of OBX animals. Previous studies have reported that glutamatergic systems, such as NMDAR1 and GluR1, are one of the molecular biological bases underlying learning and memory, as well as depression, and that glutamate receptor stimulation-triggered phosphorylation of some key protein such as NMDAR, AMPAR, CaMKII, and CREB is a molecular mechanism underlying neuroplasticity in the hippocampus $(31-33)$. Indeed, stimulation of AMPAand NMDA-type glutamate receptors increases the intracellular $\mathrm{Ca}^{2+} / \mathrm{Na}^{+}$level via the glutamate-gated cation channels on neuronal membranes, leading to the activation of calmodulin and other $\mathrm{Ca}^{2+}$-dependent enzymes, and thereby elicits phosphorylation of subunits of NMDAR and AMPAR at glutamatergic synapses via the activation of protein kinases $\mathrm{A}$ and/or C, CaMKII (32). Moreover, autophosphorylation of CaMKII triggered by $\mathrm{Ca}^{2+}$-dependent activation of calmodulin plays an important role in the conversion of short-term memory to long-term memory (18). A phosphorylated form of CREB is also known to play a role in the transcription of late downstream genes encoding proteins such as neurotrophic/growth factors which have been implicated in memory formation (33) and depression (34).

In this study, we focused on these factors for the following reasons: First, previous reports from this laboratory and others $(9,18,27)$ demonstrated that OBX deteriorates the septo-hippocampal cholinergic system and thereby induces learning and memory performance deficits. Secondly, cholinergic systems affect glutamate receptor function via the activation of muscarinic receptors coupled with Gq/11-PKC signaling systems and thereby modulate glutamatergic neurotransmission $(9$, 35, 36). As shown in Fig. 5, OBX significantly reduced the levels of p-GluR1, p-NMDAR1, p-CaMKII, and p-CREB in the hippocampus without affecting the basal expression levels of non-phosphorylated forms of these proteins. These findings agree with our previous reports (8) and indicate that cognitive deficits observed in OBX animals are at least partly due to OBX-induced dysfunction of the synaptic plasticity-related neuronal signaling system in the hippocampus. This idea is supported by the fact that OBX-induced dysfunction of the aforementioned neuronal signaling systems was significantly ameliorated in the OBX groups, the cognitive performance of which was improved by BS and tacrine treatment. Moreover, it is of interest to note that imipramine treatment attenuated only OBX-induced down- regulation of CaMKII phosphorylation in the hippocampus. Considering the data that imipramine failed to affect the cognitive dysfunction of OBX animals, it is likely that an increase in CaMKII autophosphorylation may be insufficient to induce neuroplasticity related to cognitive function.

The present study revealed that administration of tacrine and BS attenuated the OBX-induced downregulated expression level of ChAT in the hippocampus. OBX-induced decrease in the expression level of ChAT and its reversal by tacrine are indeed consistent with our previous reports $(8,10)$. Daily tacrine administrationinduced protection of septal cholinergic neurons has also been observed in an animal model of type 2 diabetes, the cognitive dysfunction of which was ameliorated by tacrine $(23,37,38)$, suggesting that elevation of endogenous acetylcholine protects cholinergic neuron from degeneration under pathological conditions. Interestingly, in this study, we found that systemic administration of BS, as well as of tacrine, inhibited the ex vivo activity of AChE in brain tissue. Therefore, a possible explanation for the effect of BS on ChAT expression in OBX animals is that BS may elevate the endogenous acetylcholine level via its tacrine-like action and thereby protect the septo-hippocampal cholinergic systems from OBX-induced neurodegenerative damage in the brain. This mechanism is also very likely to be involved in ameliorating/enhancing the effects of BS on the synaptic plasticity-related neuronal signaling system and cognitive performance in OBX animals, since evidence indicates that endogenous acetylcholine exhibits a facilitatory role in the NMDA-receptor function via $\mathrm{M}_{1}$ muscarinic receptors in the brain (36) and that the $M_{1}$ receptormediated cognitive behavior is mediated by neurosignaling pathways including CREB phosphorylation and BDNF expression and secondary messenger cascades, like $\mathrm{G}_{\mathrm{q} / 11}$-PKC signaling, and intracellular $\mathrm{Ca}^{2+}$ mobilization (36).

The present study also investigated using the TST as a model to detect depression-like behavior (39), whether BS treatment affects emotional deficits that are distinctively observed in OBX animals $(10,40)$. The vehicle-treated OBX mice showed significantly prolonged immobility time in a manner reversible by imipramine treatment in the TST. These data agree with previous studies $(10,21)$ and indicate that increased immobility of OBX animals in this test can be used as an index of a behavioral symptom relevant to depression. It should be noted that the depression-like behavior deficits caused by OBX were ameliorated in the BStreated OBX group, as well as in the imipramine-treated OBX animals. These findings raise the possibility that BS has an antidepressant-like effect. This idea seemed to 
be supported by our recent observations that BS administration exhibited imipramine-like effects in an animal model of chronic mild stress (Mizuki et al., unpublished data).

The mechanism(s) by which BS exhibits the antidepressant-like effect is unclear. The present study suggested involvement of synaptic plasticity-related signaling and central cholinergic systems in the ameliorative effects of $\mathrm{BS}$ and tacrine on OBX-induced memory deficits. However, considering the failure of tacrine to ameliorate depression-like behavior of the OBX animals, it is very likely that the anti-depressant-like effect of BS is mediated by a mechanism(s) differing from that implicated in BS-induced amelioration of cognitive deficits caused by OBX. Nevertheless, further investigation is required to clarify the exact mechanisms involved in the anti-dementia drug-like and antidepressant-like effects of BS.

It is still unclear which chemical constituents account for the anti-dementia drug-like and anti-depressant-like effects of BS observed in OBX animals. However, a speculative explanation for the effects of BS is that some chemical constituents of BS like isoflavonolignans such as butesuperin $\mathrm{A}, \mathrm{B}$, and $(-)$ medicarpin may play an important role in the behavioral and neurochemical effects of BS in OBX animals. Ma et al. (4) found that butesuperin $A$ and $B$ exerted inhibitory activities against phosphodiesterase type $3 \mathrm{~A}$ and type 5 in in vitro, and recent studies demonstrated that selective phosphodiesterase-5 inhibitors such as Sildenafil, which are used for ED therapy, ameliorated emotional deficits like depression $(41,42)$. Moreover, $(-)$ medicarpin is reportedly an activator of the neurogenin 2 promoter (43). Neurogenin2 is involved in neural differentiation and neurogenesis (44). Although no information is currently available on whether these chemicals are able to affect the ex vivo activity of $\mathrm{AChE}$ like $\mathrm{BS}$, these constituents are likely to contribute to the amelioration of cognitive dysfunction and depression-related behavior in OBX animals. This possibility needs to be examined by pharmacokinetic analysis of chemical constituents which can cross the blood-brain barrier and be detected in the brain tissue after systemic administration of BS. Nevertheless, further investigations are required to have a better understanding of the mechanism underlying the action of BS.

In summary, the present study has demonstrated that daily administration of BS ameliorates OBX-induced cognitive and emotional disturbance via different neuronal mechanisms and that the effect of BS on the cognitive function in OBX animals is mediated by facilitating the neuro-signaling system in the hippocampus and central cholinergic system.

\section{Acknowledgment}

KM reports a grant and Butea superba from Shiratori Pharmaceutical Co., Ltd.

\section{Conflicts of Interest}

The authors declare that they have no competing interests.

\section{References}

1 Suntara A. The remedy pamplet of Kwao Krua Tuber of Luang Anusarnsuntatakromkarnphiset. Chiang mai: Chiang Mai Upatipongsa Press; 1931.

2 Tocharus C, Smitasiri Y, Jeenapongsa R. Butea superba Roxb. enhances penile erection in rats. Phytother Res. 2006;20:484489.

3 Cherdshewasart W, Nimsakul N. Clinical trial of Butea superba, an alternative herbal treatment for erectile dysfunction. Asian J Androl. 2003;5:243-246.

4 Ma K, Ishikawa T, Seki H, Furihata K, Ueki H, Narimatsu S, et al. Isolation of new isoflavonolignans, butesuperins $\mathrm{A}$ and B, from a Thai miracle herb, Butea superba. Heterocycles. 2005;65:893-900.

5 Mangialasche F, Solomon A, Winblad B, Mecocci P, Kivipelto M. Alzheimer's disease: clinical trials and drug development. Lancet Neurol. 2010;9:702-716.

6 Shoji M. [Drug therapy for Alzheimer's disease]. Brain Nerve. 2010;62:777-785. (text in Japanese)

7 Oba A, Nakagawasai O, Onogi H, Nemoto W, Yaoita F, Arai Y, et al. Chronic fluvoxamine treatment changes $5-\mathrm{HT}_{2 \mathrm{~A} / 2 \mathrm{C}}$ receptor-mediated behavior in olfactory bulbectomized mice. Life Sci. 2013;92:119-124.

8 Le TX, Pham TNH, Do TP, Fujiwara H, Tanaka K, Li F, et al. Bacopa monnieri ameliorates memory deficits in olfactory bulbectomized mice: Possible involvement of glutamatergic and cholinergic systems. Neurochem Res. 2013;38:2201-2215.

9 Yamada M, Hayashida M, Zhao Q, Shibahara N, Tanaka K, Miyata T, et al. Ameliorative effects of yokukansan on learning and memory deficits in olfactory bulbectomized mice. J Ethnopharmacol. 2011;135:737-746.

10 Sithisarn P, Rojsanga P, Jarikasem S, Tanaka K, Matsumoto K. Ameliorative effects of acanthopanax trifoliatus on cognitive and emotional deficits in olfactory bulbectomized mice: an animal model of depression and cognitive deficits. Evid Based Complement Alternat Med. 2013;2013:701956.

11 Kelly JP, Wrynn AS, Leonard BE. The olfactory bulbectomized rat as a model of depression: an update. Pharmacol Ther. 1997;74:299-316.

12 Redmond AM, Kelly JP, Leonard BE. Behavioural and neurochemical effects of dizocilpine in the olfactory bulbectomized rat model of depression. Pharmacol Biochem Behav. 1997; 58:355-359.

13 Wang D, Noda Y, Tsunekawa H, Zhou Y, Miyazaki M, Senzaki $\mathrm{K}$, et al. Behavioural and neurochemical features of olfactory bulbectomized rats resembling depression with comorbid anxiety. Behav Brain Res. 2007;178:262-273.

14 Bahar-Fuchs A, Chetelat G, Villemagne VL, Moss S, Pike K, Masters CL, et al. Olfactory deficits and amyloid- $\beta$ burden in Alzheimer's disease, mild cognitive impairment, and healthy 
aging: a PiB PET study. J Alzheimers Dis. 2010;22:1081-1087.

15 Bahar-Fuchs A, Moss S, Rowe C, Savage G. Olfactory performance in $\mathrm{AD}$, aMCI, and healthy ageing: a unirhinal approach. Chemical Senses. 2010;35:855-862.

16 Wesson DW, Levy E, Nixon RA, Wilson DA. Olfactory dysfunction correlates with amyloid-beta burden in an Alzheimer's disease mouse model. J Neurosci. 2010;30:505-514.

17 Aleksandrova IY, Kuvichkin VV, Kashparov IA, Medvinskaya NI, Nesterova IV, Lunin SM, et al. Increased level of betaamyloid in the brain of bulbectomized mice. Biochemistry (Mosc). 2004;69:176-180.

18 Hozumi S, Nakagawasai O, Tan-No K, Niijima F, Yamadera F, Murata A, et al. Characteristics of changes in cholinergic function and impairment of learning and memory-related behavior induced by olfactory bulbectomy. Behav Brain Res. 2003;138:9-15.

19 Horai H, Arita M, Kanaya S, Nihei Y, Ikeda T, Suwa K, et al. MassBank: a public repository for sharing mass spectral data for life sciences. J Mass Spectrom. 2010;45:703-714.

20 Zhao Q, Murakami Y, Tohda M, Obi R, Shimada Y, Matsumoto K. Chotosan, a Kampo formula, ameliorates chronic cerebral hypoperfusion-induced deficits in object recognition behaviors and central cholinergic systems in mice. J Pharmacol Sci. 2007; 103:360-373.

21 Shioda N, Yamamoto Y, Han F, Moriguchi S, Yamaguchi Y, Hino M, et al. A novel cognitive enhancer, ZSET1446/ST101, promotes hippocampal neurogenesis and ameliorates depressive behavior in olfactory bulbectomized mice. J Pharmacol Exp Ther. 2010;333:43-50.

22 Inada C, Thi Le X, Tsuneyama K, Fujiwara H, Miyata T, Matsumoto K. Endogenous acetylcholine rescues NMDAinduced long-lasting hippocampal cell damage via stimulation of muscarinic $\mathrm{M}(1)$ receptors: elucidation using organic hippocampal slice cultures. Eur J Pharmacol. 2013;699:150-159.

23 Zhao Q, Matsumoto K, Tsuneyama K, Tanaka K, Li F, Shibahara $\mathrm{N}$, et al. Diabetes-induced central cholinergic neuronal loss and cognitive deficit are attenuated by tacrine and a Chinese herbal prescription, kangen-karyu: elucidation in type 2 diabetes $\mathrm{db} / \mathrm{db}$ mice. J Pharmacol Sci. 2011;117:230-242.

24 Ellman GL, Courtney KD, Andres V Jr, Feather-Stone RM. A new and rapid colorimetric determination of acetylcholinesterase activity. Biochem Pharmacol. 1961;7:88-95.

25 Bertaina-Anglade V, Enjuanes E, Morillon D, Drieu la Rochelle C. The object recognition task in rats and mice: a simple and rapid model in safety pharmacology to detect amnesic properties of a new chemical entity. J Pharmacol Toxicol Methods. 2006;54:99-105.

26 De Rosa R, Garcia AA, Braschi C, Capsoni S, Maffei L, Berardi $\mathrm{N}$, et al. Intranasal administration of nerve growth factor (NGF) rescues recognition memory deficits in $\mathrm{AD} 11$ anti-NGF transgenic mice. Proc Natl Acad Sci U S A. 2005;102:3811-3816.

27 Saunders NL, Summers MJ. Attention and working memory deficits in mild cognitive impairment. J Clin Neuropsychol. 2009;32:350-357.

28 Csernansky JG, Martin M, Shah R, Bertchume A, Colvin J, Dong H. Cholinesterase inhibitors ameliorate behavioral deficits induced by MK-801 in mice. Neuropsychopharmacology. 2005;
30:2135-2143.

29 Bliss TV, Collingridge GL. A synaptic model of memory: longterm potentiation in the hippocampus. Nature. 1993;361:31-39.

30 Neves G, Cooke SF, Bliss TV. Synaptic plasticity, memory and the hippocampus: a neural network approach to causality. Nat Rev Neurosci. 2008;9:65-75.

31 Zhao H, Li Q, Pei X, Zhang Z, Yang R, Wang J, et al. Long-term ginsenoside administration prevents memory impairment in aged C57BL/6J mice by up-regulating the synaptic plasticity-related proteins in hippocampus. Behav Brain Res. 2009;201:311-317.

32 Chen BS, Roche KW. Regulation of NMDA receptors by phosphorylation. Neuropharmacology. 2007;53:362-368.

33 Lamprecht R. CREB: a message to remember. Cell Mol Life Sci. 1999;55:554-563.

34 Alboni S, Tascedda F, Corsini D, Benatti C, Caggia F, Capone $\mathrm{G}$, et al. Stress induces altered CRE/CREB pathway activity and BDNF expression in the hippocampus of glucocorticoid receptor-impaired mice. Neuropharmacology. 2011;60:1337-1346.

35 Drever BD, Riedel G, Platt B. The cholinergic system and hippocampal plasticity. Behav Brain Res. 2011;221:505-514.

36 Calabresi P, Centonze D, Gubellini P, Pisani A, Bernardi G. Endogenous ACh enhances striatal NMDA-responses via M1like muscarinic receptors and PKC activation. Eur J Neurosci. 1998;10:2887-2895.

37 Zhao Q, Niu Y, Matsumoto K, Tsuneyama K, Tanaka K, Miyata $\mathrm{T}$, et al. Chotosan ameliorates cognitive and emotional deficits in an animal model of type 2 diabetes: possible involvement of cholinergic and VEGF/PDGF mechanisms in the brain. BMC Complement Altern Med. 2012;12:188.

38 Matsumoto K, Zhao Q, Niu Y, Fujiwara H, Tanaka K, SasakiHamada S, et al. Kampo formulations, chotosan, and yokukansan, for dementia therapy: existing clinical and preclinical evidence. J Pharmacol Sci. 2013;122:257-269.

39 Cryan JF, Mombereau C. In search of a depressed mouse: utility of models for studying depression-related behavior in genetically modified mice. Mol Psychiatry. 2004;9:326-357.

40 Harkin A, Kelly JP, McNamara M, Connor TJ, Dredge K, Redmond A, et al. Activity and onset of action of reboxetine and effect of combination with sertraline in an animal model of depression. Eur J Pharmacol. 1999;364:123-132.

41 Dadomo H, Parmigiani S, Nicolini Y, Freschini S, Gioiosa L, Patrelli TS, et al. Repeated and chronic administration of Vardenafil or Sildenafil differentially affects emotional and socio-sexual behavior in mice. Behav Brain Res. 2013;253: 103-112.

42 Matsushita H, Matsuzaki M, Han XJ, Nishiki TI, Ohmori I, Michiue $\mathrm{H}$, et al. Antidepressant-like effect of sildenafil through oxytocin-dependent cyclic AMP response element-binding protein phosphorylation. Neuroscience. 2012;200:13-18.

43 Arai MA, Koryudzu K, Koyano T, Kowithayakorn T, Ishibashi M. Naturally occurring Ngn2 promoter activators from Butea superba. Mol Biosyst. 2013;9:2489-2497.

44 Matsumoto M, Imura T, Fukazawa T, Sun Y, Takeda M, Kajiume $\mathrm{T}$, et al. Electrical stimulation enhances neurogenin2 expression through beta-catenin signaling pathway of mouse bone marrow stromal cells and intensifies the effect of cell transplantation on brain injury. Neurosci Lett. 2013;533:71-76. 